\title{
„Da baut sich was auf, da ist jemand, der ist verlässlich da, der hat auch Geld" - Akteurskonstellationen, Handlungskoordination und Leistungen in fragilen organisationalen Strukturen am Beispiel der arbeitsorientierten Grundbildung
}

\author{
Julia Koller (D) · Jana Arbeiter • Michael Schemmann
}

Eingegangen: 21. Dezember 2020 / Überarbeitet: 25. Februar 2021 / Angenommen: 15. April 2021 /

Online publiziert: 10. Mai 2021

(C) Der/die Autor(en) 2021

Zusammenfassung Der Beitrag untersucht anhand der theoretischen Ansätze der Educational Governance und des Neo-Institutionalismus die Steuerung in dem, bezüglich Finanzierung und Angebotsentwicklung fragilen Feld der arbeitsorientierten Grundbildung. Dabei wird fokussiert, wie soziale Ordnungen über die Koordination von Handlungen in verschiedenen Akteurskonstellationen pädagogische Leistung möglich machen. Konkret geht es darum, herauszuarbeiten, welche institutionellen und organisationalen Strukturen in der arbeitsorientierten Grundbildung erkennbar sind und welche Formen der Handlungskoordination zwischen Akteuren welche pädagogischen Leistungen zustande kommen lassen. Empirische Befunde weisen auf ein differenziertes und unterschiedlich dichtes Mehrebenensystem hin, in dem die Akteure über unterschiedliche Formen der Handlungskoordination Leistungen sichern und weiterentwickeln.

Schlüsselwörter Steuerung in der Erwachsenenbildung · Akteurskonstellationen · Handlungskoordination · Grundbildung

Dr. J. Koller $(\bowtie) \cdot$ J. Arbeiter · Prof. Dr. M. Schemmann

Universität zu Köln, Köln, Deutschland

E-Mail: julia.koller@uni-koeln.de

J. Arbeiter

E-Mail: Jana.arbeiter@uni-koeln.de

Prof. Dr. M. Schemmann

E-Mail: michael.schemmann@uni-koeln.de 


\title{
"Something is building up, there is someone who is reliable and who has money" - Actor constellations, coordination of action and educational output in fragile organizational settings. The example of work-oriented basic education
}

\begin{abstract}
The paper focuses on governance in the field of work-oriented basic education, seen as fragile with regard to funding and output, using the theoretical approaches of educational governance and neo-institutionalism. The focus is on how social orders make educational output possible through the coordination of actions in different constellations of actors. The article asks which institutional and organizational structures in work-oriented basic education can be identified, which modes of coordination of action between actors, and which outcome is achieved. Empirical findings point to a differentiated and variously dense multi-level system in which the actors coordinate their actions in different ways to secure and develop performance.
\end{abstract}

Keywords Educational Governance - Constellation of actors · Forms of coordination of action · Adult Basic Education

\section{Einleitung}

Fragen der Steuerung der Weiterbildung haben die Weiterbildungsforschung seit Beginn der Begründung als Wissenschaftsdisziplin beschäftigt. Dabei dominierte auch in der Weiterbildungsforschung über lange Zeit die allenthalben vorherrschende Vorstellung der direkten Steuerung durch den Staat. Sämtliche Ansätze und Modelle, die seit Ende des Zweiten Weltkriegs in verschiedenen wissenschaftlichen Disziplinen zur Erklärung von Steuerung vorgelegt wurden, folgten dieser ,,top down or legislator's perspective“ (Mayntz 1998, S. 15). Gegen Ende der 1980er und frühen 1990er Jahre nährte sich in der Politikwissenschaft indes grundlegender Zweifel an einem Steuerungsverständnis, das nurmehr Akteure an der Spitze fokussiert, denen umfassende Macht zugeschrieben wird.

Dieser Zweifel fand auch in der Weiterbildungsforschung Resonanz.

Angesichts wachsender Einsicht in die Mehrstufigkeit und Sektorspezifität politischer Entscheidungsprozesse musste die machiavellistische Vorstellung staatlicher einliniger Führung durch den Machtstaat ebenso als unterkomplex aufgegeben werden wie das liberalistische Konzept des Nachtwächterstaates (Faulstich und Haberzeth 2015, S. 264f.).

Mit dem Sammelband „Steuerung und Organisation in der Weiterbildung“ aus dem Jahr 2008 (Hartz und Schrader 2008) begann eine intensive Debatte und eine Auseinandersetzung mit Fragen der Steuerung in der Weiterbildung (Hof et al. 2011). Dabei werden in der Weiterbildungsforschung auch theoretische Ansätze aufgenommen, die Steuerung in der Mehrebenenperspektive betrachten, so wie etwa der Ansatz der Educational Governance (Euringer 2016; Schemmann 2020). 
Die Perspektive der Educational Governance wendet sich der Frage zu, wie soziale Ordnungen einerseits und Leistungen andererseits in Bildungssystemen hergestellt werden. Die Perspektive wurde zunächst in der Schulforschung entwickelt und entfaltet (Altrichter et al. 2007) und hat sich hier seither als ausgesprochen produktiver Analyseansatz erwiesen. In der Weiterbildungsforschung wird die Governance-Perspektive mittlerweile ebenfalls für empirische Untersuchungen verwendet. Zuvörderst ist hier das DFG-Projekt „Governance-Strukturen und pädagogische Leistungsprofile in Organisationen der Weiterbildung (GLOW)“ (SCHE 585/2-1; DO 746/3-1) zu nennen, in dem Akteurskonstellationen und Koordinationsformen in Volkshochschulen sowie die organisationsspezifischen Leistungsprofile untersucht worden sind (Schemmann 2020). Der große Vorzug der Perspektive besteht darin, die Leistungen von Weiterbildungsorganisation als Ergebnis der Koordination einer Vielzahl von Akteuren zu betrachten, die in interdependenten Abhängigkeiten stehen.

Blickt man auf die Untersuchungen aus der Perspektive der Educational Governance in Hochschul-, Schul- und Weiterbildungsforschung insgesamt, so fällt auf, dass diese Untersuchungen Einrichtungen mit finanziell gesicherten institutionellen Strukturen in den Blick nehmen, in denen sich ein institutioneller Wandel vollzieht oder vollziehen soll. Dies ist umso bemerkenswerter, als in der Weiterbildungsforschung der Ansatz noch nicht genutzt wurde, um Bereiche, in denen keine festen institutionellen und organisationalen Strukturen bestehen, zu untersuchen.

Einen solchen Bereich stellt die arbeitsorientierte Grundbildung (AoG) dar. Diese soll im Folgenden in den Fokus gerückt werden. AoG wird als komplexe Bildungsdienstleistung im Sinne einer ,,aufsuchenden Strategie - Bildungsmöglichkeiten in Alltags- und Lebensräumen zu schaffen“, verstanden (Schwarz 2021, S. 17). Dabei genießt sie derzeit, nicht zuletzt im Kontext der AlphaDekade, hohe gesellschaftliche Bedeutung und ist zugleich durch Fragilität und ständigen Wandel im Hinblick auf Finanzierung, thematische Ausrichtung und Aufgaben sowie Akteuren (wie bspw. Unternehmen, verschiedene Sozialpartner in unterschiedlichen Branchen) zu kennzeichnen. Zu verweisen ist auf ein Bedingungsgefüge zwischen betrieblicher und allgemeiner Bildung mit vielfältigen Akteuren und einer fragilen Finanzierung (Koller et al. 2020). ${ }^{1}$ Der mittlerweile erarbeitete Forschungsstand ist bemerkenswert und fokussiert Konzepte, Maßnahmen von Beratung und Schulung von Akteuren der Arbeitswelt sowie Professionalisierung der Lehrenden und hat nicht zuletzt vom BMBF-Förderschwerpunkt ,Arbeitsplatzorientierte Alphabetisierung und Grundbildung Erwachsener“ (2012-2015; Kowalczyk et al. 2016) sowie der derzeit laufenden AlphaDekade maßgeblich profitiert (BMBF und Kultusministerkonferenz 2016; Schroeder 2016; Lernende Region - Netzwerk Köln e.V. 2021).

Hohe praktische Relevanz erfährt die Frage nach Steuerung im Feld der AoG durch Fragen der Verstetigung und der Entwicklung neuer Strukturen im Zusammenhang mit bildungspolitischen Maßnahmen (wie bspw. der Nationalen Dekade

\footnotetext{
${ }^{1}$ Die hier vorgestellte Studie steht in Zusammenhang mit der Verlängerung des Projekts ABAG ${ }^{2}$ von Mai 2019-Juli 2020. Das Projekt $\mathrm{ABAG}^{2}$ beschäftigte sich zwischen 2016 und 2019 mit Gelingensbedingungen, Effekten der Teilnahme und Professionalisierung der AoG. In der aktuellen zweiten Verlängerung (August 2020-August 2021) steht die Entwicklung eines Leitfaden für den mikrodidaktischen Planungsprozess in der AoG im Fokus. Finanziert wird das Projekt mit dem Förderkennzeichen W142400B vom Bundesministerium für Bildung und Forschung im Rahmen der AlphaDekade.
} 
für Alphabetisierung und Grundbildung). So wird davon ausgegangen, dass ,nur dann nachhaltige Wirkungen für die Alphabetisierung und Grundbildung erzielt werden [können], wenn an vorhandene Strukturen angeknüpft" wird (BMBF und Kultusministerkonferenz 2016). Welche Strukturen und Akteurskonstellationen zur Leistungserbringung von AoG vorhanden sind und welche Formen der Handlungskoordination in diesen fragilen Settings sichtbar werden, ist allerdings noch nicht untersucht. Entsprechend werden in diesem Beitrag Erkenntnisse darüber generiert,

- welche institutionellen und organisationalen Strukturen sich aktuell erkennen lassen und

- welche Formen der Handlungskoordination zwischen Akteuren sowie welche pädagogischen Leistungen zustande kommen (DiMaggio und Powell 2009; Herbrechter und Schemmann 2019).

Zur Beantwortung der Fragestellungen gehen wir so vor, dass wir zunächst die Perspektive der Educational Governance sowie einzelne Elemente des soziologischen Institutionalismus als theoretischen Rahmen näher erörtern, bevor das methodische Design gekennzeichnet wird. Zur Beantwortung der Fragen wurde ein zweistufiges methodisches Vorgehen gewählt. Daran schließt sich die Darstellung sowie die Diskussion der Befunde an, ehe der Beitrag in einem Fazit die Einordnung der Befunde in die Steuerungsdebatte in der Erwachsenen- und Weiterbildung vornimmt und weiteren Forschungsbedarf kennzeichnet.

\section{Educational Governance und Neo-Institutionalismus als theoretische Rahmung}

Als theoretischer Bezugspunkt der Untersuchung werden der Ansatz der Educational Governance und Elemente des Neo-Institutionalismus herangezogen.

Als Forschungsansatz der Bildungsforschung untersucht die Governance-Perspektive das Zustandekommen, die Aufrechterhaltung und die Transformation von Leistungen im Bildungswesen unter der Perspektive der Handlungskoordination zwischen verschiedenen Akteuren in komplexen Mehrebenensystemen (Maag Merki und Altrichter 2010, S. 22).

Im Kern des Interesses der analytischen Perspektive steht die Koordination von Handlung (Altrichter 2015). In den Blick rückt das Management von Interdependenzen kollektiver und individueller Akteure.

Des Weiteren wird eine Vielzahl von Akteuren und Akteurskonstellationen berücksichtigt. Das Handeln der Akteure basiert auch immer auf Institutionen bzw. einem institutionellen System von Regeln. Dadurch wird Sicherheit in Entscheidungsprozessen gewährleistet.

Auch die Betrachtung von sozialen Systemen in der Mehrebenenperspektive gehört zu den Kennzeichen. Und schließlich sind auch Misch- und Hybridformen der Handlungskoordination als konstitutiv zu nennen. Damit wird darauf verwiesen, dass unterschiedliche Formen der Handlungskoordination nicht nur nebeneinander existieren, sondern auch interagieren und einander gegenseitig beeinflussen. 
Im Folgenden sollen die Formen der Handlungskoordination (Hierarchie, Netzwerk, Gemeinschaft, Markt) näher gekennzeichnet werden:

- hierarische Handlungskoordinationsformen,

- netzwerkliche Handlungskoordinationsformen,

- gemeinschaftliche Handlungskoordinationsformen,

- Handlungskoordinationsformen des Marktes.

Sie dienen in empirischen Zusammenhängen als idealtypische Analysemittel und beschreiben institutionell verdichtete, komplexe Formen der Koordination, die auf Mechanismen der Beobachtung, Beeinflussung und Verhandlung sowie den Verfügungsrechten beruhen (Kussau und Brüsemeister 2007, S. 40).

Spricht man von hierarchischen Handlungskoordinationsformen, kann davon ausgegangen werden, dass die Entscheidungsbefugnis bei einer übergeordneten Leistungsinstanz liegt (vgl. ebd., S. 41). Die kollektive Handlungsfähigkeit wird dadurch maßgeblich bestimmt.

Im Rahmen von netzwerklichen Handlungskoordinationsformen wird kollektive Handlungsfähigkeit dann erlangt, wenn auf der Grundlage von wechselseitiger Beeinflussung Informationsvermittlung und Ressourcentausch entstehen. Hierbei stehen die Akteure nicht in einem über- oder untergeordneten Verhältnis (Benz und Dose 2010, S. 262ff.).

Gemeinschaftliche Handlungskoordinationsformen sind geprägt durch affektives Handeln und eine starke Bindung der Akteure untereinander, die durch geteilte kognitive Überzeugungen und internalisierte Normen entsteht. Diese beruht auf der Historie der Gemeinschaft, wodurch sie als Ergebnis eingeübter Interaktionspraxis zu verstehen ist (Benz und Dose 2010, S. 257).

Im Rahmen der Handlungskoordinationsform des Marktes steht die Beobachtung der einzelnen Akteure im Vordergrund, wobei die zur Verfügung stehenden Ressourcen eine bedeutsame Rolle spielen (vgl. ebd.).

Um regionalen und kulturellen Gegebenheiten gerecht $\mathrm{zu}$ werden, besteht der Kern der Analyse der Educational Governance auch darin, das Governance-Regime als eine Mischform der Handlungskoordinationsformen im Gesamtzusammenhang zu verstehen und zu identifizieren (Kussau und Brüsemeister 2007, S. 41). Governance-Regimes kennzeichnen also den Zusammenhang der unterschiedlichen Formen der Handlungskoordination. In diesem Verständnis unterliegt das Governance-Regime einem ständigen Wandel, der unter anderem durch bildungspolitische Diskurse und Programme intendiert oder nicht-intendiert beeinflusst wird. Somit rücken auch bildungspolitische Maßnahmen als Gegenstand der Educational-GovernanceForschung in den Vordergrund (vgl. ebd.).

Dass sich die Perspektive der Educational Governance mit dem Neo-Institutionalismus in produktiver Weise verbinden lässt, ist an verschiedenen Stellen herausgearbeitet worden (u. a. Herbrechter und Schemmann 2019). In diesem Zusammenhang soll vor allem auf den Begriff ,organisationales Feld“ aus dem Neo-Institutionalismus abgestellt werden.

Organisationen sind im Verständnis des Neo-Institutionalismus keine geschlossenen Einheiten, sondern offene Systeme, die im Austausch mit der Umwelt ste- 
hen (Koch und Schemmann 2009). Jede Organisation ist laut DiMaggio und Powell (2009) Teil eines organisationalen Feldes. Dabei bezeichnet das organisationale Feld

jene Organisationen, die als Aggregat einen erkennbaren Bereich des institutionellen Lebens konstituieren: Zentrale Lieferanten, Konsumenten von Ressourcen und Produkten, Regulierungsbehörden und andere Organisationen, die ähnliche Dienstleistungen oder Produkte herstellen (ebd., S. 59).

Die Strukturierung des Feldes unterscheidet sich laut DiMaggio und Powell (2009) in der Abhängigkeit von folgenden Aspekten:

die Zunahme des Umfangs von Interaktionen zwischen den Organisationen des Feldes; die Ausbildung scharf definierter interorganisationaler Herrschaftsstrukturen und Koalitionsmuster; eine Zunahme der Informationsmenge, mit der sich die Organisationen eines Feldes auseinandersetzen müssen; und die Entwicklung einer gegenseitigen Wahrnehmung unter den Teilnehmern eines Sets von Organisationen, dass sie an einer gemeinsamen Unternehmung beteiligt sind (ebd., S. 60).

Mit dem Forschungsfokus auf Fragen der Handlungskoordination zwischen den Akteuren lässt sich mit der Perspektive des Neo-Institutionalismus erweitert fragen, „ob sich Formen der Handlungskoordination zwischen Akteuren in Abhängigkeit vom Strukturierungsgrad verändern“ (Herbrechter und Schemmann 2019, S. 188).

In diesem Abschnitt wurden die theoretischen Grundannahmen erläutert. Wie sich diese in dem Feld der AoG ausgestalten, ist Bestandteil der empirischen Untersuchung.

\section{Methodisches Vorgehen}

Im Folgenden wird das mehrstufige methodische Vorgehen erläutert. Den ersten Schritt stellt eine systematische kategorienbasierte Internetrecherche dar, durch die eine bundesweite Übersicht über die Akteure und die pädagogischen Leistungen in der AoG erarbeitet wird. Auf dieser empirischen Grundlage werden zunächst Muster der organisationalen Struktur von AoG identifiziert und sodann orientiert an diesen Mustern drei Fälle für die multiple case study ausgewählt (Yin 2009, S. 39). Im Anschluss erfolgt die Datenerhebung in den ausgewählten Fällen mittels Experteninterviews sowie die Auswertung und Analyse der Daten.

In Unterkapitel 3.1 wird zunächst das Vorgehen der systematischen Internetrecherche erläutert, die empirische Ergebnisse an sich hervorgebracht hat, zugleich aber die Grundlage für die Fallauswahl darstellt. In Unterkapitel 3.2 folgt die Erörterung des multiple case study-Designs mitsamt Datenerhebung und Datenauswertung.

\subsection{Systematische kategorienbasierte Internetrecherche}

Das Ziel der systematischen kategorienbasierten Internetrecherche besteht darin, organisationale Strukturen, Akteure sowie Leistungen der AoG bundesweit sichtbar zu machen. Auf der Grundlage der Internetrecherche konnten 127 Angebote, Projekte 

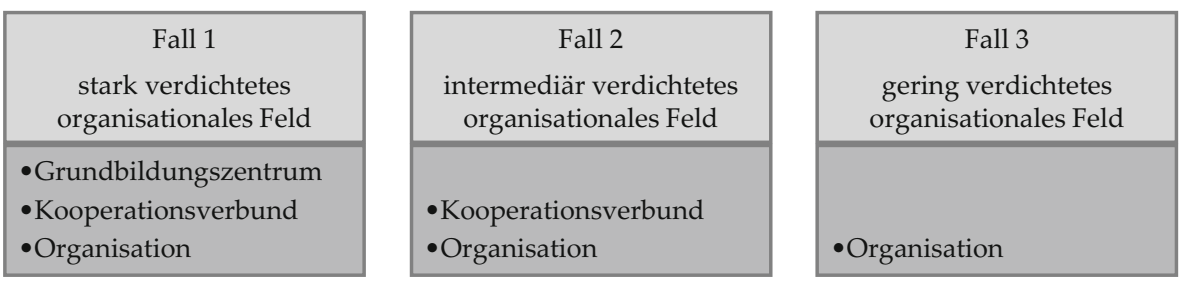

\section{Leistungsebene}

Abb. 1 Fallkonstruktion: Dichte des organisationalen Feldes

oder Initiativen unterschiedlicher Fördergeber der AoG identifiziert werden. Diese wurden entlang von Strukturmerkmalen, wie Finanzierung, Ziele usw., gekennzeichnet und analysiert. Die systematisierten Auswertungen und Übersichten wurden den für AoG zuständigen Referaten der Kultusministerien der entsprechenden Bundesländer vorgelegt. Die Expertise und Feldkenntnis in den Referaten ermöglichten eine Validierung und Ergänzung der Übersichten. Ferner wurden Kurzberichte erstellt, die Aufschluss über die strukturellen Besonderheiten der AoG im jeweiligen Bundesland geben und Vergleiche ermöglichen. Die Ergebnisse der systematischen kategorienbasierten Internetrecherche erlauben es, Akteure der AoG in einem Mehrebenensystem (Schrader 2011) darzustellen sowie Muster der organisationalen Strukturen zu identifizieren. Hierbei wird sichtbar, dass regionale Unterschiede in der Ausprägung der organisationalen Strukturen bestehen. ${ }^{2}$

Für die multiple case study werden auf der Grundlage der identifizierten Muster der organisationalen Struktur drei idealtypische Fälle ausgewählt, die eine variierende organisationale Dichte aufweisen (vgl. Abb. 1). Fall 1 kennzeichnet hierbei ein stark verdichtetes organisationales Feld, in dem sowohl Grundbildungszentren, ein Kooperationsverbund und Organisationen agieren. Fall 2 steht für ein intermediär verdichtetes organisationales Feld, in dem ein Kooperationsverbund und Organisationen vorhanden sind, während in Fall 3 lediglich verschiedene Organisationen in die Leistungserbringung von AoG eingebunden sind. Im Sinne des Ansatzes der Educational Governance haben die Koordinationsformen einen Einfluss auf die pädagogische Leistung, die hier nach Fällen unterschiedlicher organisationaler Dichte differenziert werden.

\subsection{Multiple case study}

Basierend auf der skizzierten Fallauswahl wird nun das methodische Vorgehen der multiple case study genauer beschrieben. Zur Datenerhebung der integrierten multiple case study (Yin 2009, S. 39) werden leitfadengestützte Experteninterviews ( $n=6$ ) durchgeführt. Der Aufbau der Studie folgt der Klassifizierung unterschiedlicher Fall-

\footnotetext{
${ }^{2}$ Die Ergebnisse der strukturierten kategorienbasierten Internetrecherche werden in Abschnitt 4.1. genauer dargestellt und an dieser Stelle nur kurz erwähnt, da sie entscheidend für das weitere methodische Vorgehen sind.
} 
studientypen nach Yin (vgl. ebd.). Werden demnach innerhalb eines Falls verschiedene Analyseobjekte (Grundbildungszentrum, Kooperationsverbund und Organisation) betrachtet, bezeichnet man den Fallstudienaufbau als integriert. Die Kombination mehrerer integrierter Fälle stellt schließlich ein integriertes multiple case study-Design dar. Gemäß dem regelgeleiteten und strukturierten Vorgehen ermöglicht die Fallstudie nach Yin (2009) sowohl Einzelfallanalysen als auch eine gesamtanalytische Zusammenschau und einen Fallvergleich. Pro Analyseobjekt wurde eine Expertin bzw. ein Experte ausgewählt, mit der bzw. mit dem ein leitfadengestütztes Interview geführt wurde. Als Expertinnen und Experten werden Akteure verstanden, die als Mitglied des jeweiligen Analyseobjektes gelten und dementsprechend Expertise besitzen. Konkret wurden ausschließlich Projekt- oder Programmleitungen interviewt. Für Fall 1 wurden drei Interviews, für Fall 2 zwei Interviews und Fall 3 ein Interview geführt, wobei anhand des Leitfadens die Themenbereiche Aufgaben, Ziele, Zusammenarbeit und Finanzierung angesprochen wurden.

Zur Auswertung wird die strukturierende Methode der qualitativen Inhaltsanalyse nach Mayring (2015) angewendet. Auf den Grundannahmen der Educational Governance basierend, rücken folgende Analyseeinheiten in den Fokus der Analyse:

- die pädagogische Leistung,

- Akteurskonstellationen und

- Handlungskoordinationsformen.

Neben mehreren Kontrolldurchgängen bezüglich der Eignung des Kategoriensystems wurde eine Berechnung der Intercoder-Reliabilität vorgenommen (Krippendorff 2004). Als ein wesentliches Qualitätskriterium im Rahmen empirischer Sozialforschung gibt diese darüber Auskunft, inwiefern zwei unabhängige Kodiererinnen und Kodierer mittels desselben Kategoriensystems und dem zugehörigen Codebuch zu übereinstimmenden Ergebnissen gelangen. Es wurde ein sehr guter Reliabilitätskoeffizient von 0,91 erzielt.

\section{Ergebnisse}

Im Folgenden werden die Befunde der Untersuchung dargestellt und erläutert. Zunächst stehen die Ergebnisse der strukturierten Internetrecherche sowie deren Systematisierung und Auswertung im Blick (4.1). Daran schließen die Ergebnisse der Einzelfallanalysen an, wobei hier die Handlungskoordination sowie das Leistungsspektrum im Blickpunkt stehen (4.2). Der dritte Abschnitt befasst sich schließlich mit der Auswertung des Fallvergleichs und einer Zusammenführung der Ergebnisse zu Governance Regimes der AoG (4.3).

\subsection{Arbeitsorientierte Grundbildung im Mehrebenensystem}

Die Ergebnisse der strukturierten kategorienbasierten Internetrecherche weisen darauf hin, dass vielfältige Akteure, mannigfaltige Finanzierungsstrukturen sowie verschieden ausgeprägte organisationale Strukturen zu identifizieren sind. In diesem Abschnitt wird diese Vielfalt näher beleuchtet und sodann in einer Mehrebenen- 
Abb. 2 AoG im Mehrebenensystem. (In Anlehnung an Schrader 2011)

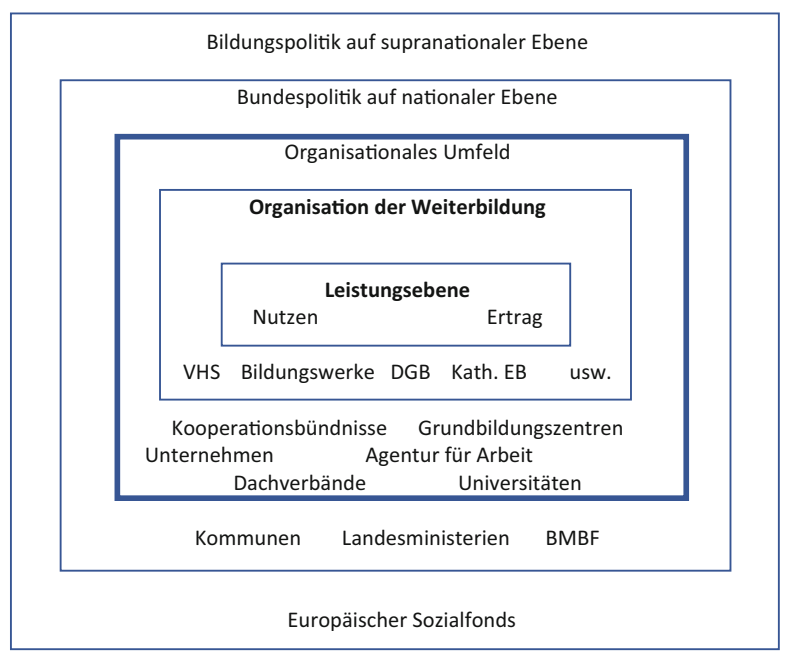

struktur systematisiert (vgl. Abb. 2). Sodann wird auch auf regionale Unterschiede eingegangen.

Richtet man den Blick auf die Frage, welche Akteure insgesamt an der Leistungserbringung von AoG beteiligt sind, so sind neben den Volkshochschulen und weiteren Bildungseinrichtungen bundesweit auch Verbände und gemeinnützige Vereine festzustellen. AoG wird also von diversen Organisationen der Weiterbildung über Projektmittel im Rahmen von Angeboten und Programmen ermöglicht. Im direkten organisationalen Umfeld lassen sich Dachverbände und Koordinierungsstellen verzeichnen. Außerdem wird AoG in implementierten Grundbildungszentren realisiert, die maßgeblich den Zweck der Koordinierung von Angeboten, Trägern und Teilnehmenden verfolgen. Sodann wird AoG im Rahmen von Kooperationsbündnissen zu einem verstetigten Thema, indem sich diverse Akteure im festen Rahmen zusammenfinden, um den Auf- und Ausbau (bestehender) Bildungsangebote voranzutreiben. Auch Universitäten als wissenschaftliche Akteure und die Agentur für Arbeit sowie Unternehmen werden im organisationalen Umfeld sichtbar. Politische Akteure werden auf der Bundesebene (BMBF) ersichtlich, in länderspezifische Ministerien und Kommunen. Der Europäische Sozialfonds (ESF) wird auf der Ebene der supranationalen Akteure bedeutsam. Die Akteursvielfalt in der AoG wird in Abb. 2 systematisch dargestellt. ${ }^{3}$

Blickt man nun auf regionale Unterschiede in den Ausprägungen der Akteure, so lassen sich diese vor allem in Bezug auf Finanzierungsstrukturen und die Ausprägung des organisationalen Umfeldes feststellen. So greifen einige Bundesländer, wie etwa Rheinland-Pfalz und Hessen, zur Finanzierung von Angeboten und Projekten sowohl auf Bundesmittel des BMBF im Rahmen der AlphaDekade als auch auf ESF-Mittel im Rahmen des Förderprogramms Alphabetisierung und Grundbildung

\footnotetext{
3 Die Darstellung der Ergebnisse der strukturierten kategorienbasierten Internetrecherche wurde im November 2019 erarbeitet. Eventuelle, danach eingetretene Veränderungen wurden nicht berücksichtigt.
} 
und auf die jeweils zur Verfügung gestellten Landesmittel zurück. Hingegen spielen in anderen Bundesländern, wie Baden-Württemberg und Berlin, vor allem Bundesund Landesmittel oder aber Bundes- und ESF-Mittel, wie in Sachsen-Anhalt, eine Rolle. Weiterhin gibt es Bundesländer, Thüringen und Saarland, in denen finanzielle Rahmenbedingungen durch spezifische Weiterbildungsgesetze geregelt sind. Darüber hinaus weisen die Ergebnisse auf Bundesländer, wie Schleswig-Holstein und Mecklenburg-Vorpommern, hin, in denen das AoG-Angebot lediglich durch Bundesmittel realisiert wird. Die bestehenden Finanzierungsstrukturen sind von begrenzter Laufzeit und somit als fragil zu kennzeichnen.

Blickt man auf das organisationale Umfeld, so weisen die Ergebnisse auf folgende Differenzen hin: In einigen Bundesländern, wie in Niedersachsen, Thüringen, Rheinland-Pfalz, sind an der Realisierung von AoG Angeboten sowohl die Organisationen der Weiterbildung beteiligt als auch die Grundbildungszentren und ein Kooperationsverbund. Das organisationale Feld ist hierbei also als stark verdichtet $\mathrm{zu}$ verstehen. Gleichzeitig gibt es Bundesländer, wie Hessen und NordrheinWestfalen, in denen Kooperationsverbünde und Organisationen der Weiterbildung aktiv sind. Hier kann von einem intermediär verdichteten organisationalen Feld gesprochen werden. In weiteren Ländern sind schließlich einzelne Organisationen der Weiterbildung für die Leistungserbringung verantwortlich, ohne, dass das organisationale Feld zusätzlich durch Kooperationsverbünde oder Grundbildungszentren geprägt ist.

Es konnte gezeigt werden, welche vielfältigen Akteure in die Leistungserbringung der AoG eingebunden sind und dass die organisationalen Strukturen regionale Differenzen aufweisen. Im Folgenden ist zu untersuchen, ob und wie sich in den verschiedenen organisationalen Strukturen auch Formen der Handlungskoordination und pädagogische Leistung unterscheiden. Dazu werden Fallstudien ausgewertet, die vor dem Hintergrund der oben gekennzeichneten Muster der organisationalen Strukturen der AoG ausgewählt wurden.

\subsection{Handlungskoordination und pädagogische Leistung in der AOG}

In diesem Abschnitt werden die Ergebnisse der Fallstudien im Hinblick auf die Formen der Handlungskoordination zwischen den Akteuren sowie die pädagogische Leistung in der AoG diskutiert. Der Analyse liegt die Annahme zugrunde, dass die Leistungserbringung in den Fällen je nach organisationaler Struktur variiert. Die Ergebnisse der untersuchten Fälle werden zunächst einzeln in ihrer jeweiligen Leistungsstruktur und in den jeweils dominierenden Formen der Handlungskoordination (Netzwerk, Gemeinschaft, Hierarchie und Markt) dargestellt. Ein Fokus wird auf die Darstellung des Erhalts und der Entwicklung pädagogischer Leistungen in einer fragilen Finanzierungssituation und der Handlungskoordination in Konstellationen mit politischen Akteuren gelegt.

\subsubsection{Fall 1}

Der Fall 1 ist durch ein stark verdichtetes organisationales Feld gekennzeichnet. Es wird ein Fall untersucht, in dem sowohl Grundbildungszentren, ein Kooperati- 
onsverbund und Organisationen in die Leistungserbringung von AoG eingebunden sind. Im Rahmen der Finanzierung der drei organisationalen Strukturen von AoG sind sowohl ESF-, Bundes- als auch Landes- und kommunale Mittel bedeutsam. Der Grad der Verdichtung des organisationalen Feldes spiegelt sich sowohl in der Vielfalt der Akteure als auch in der Breite des Leistungsspektrums wider.

Die Ausprägung der Leistungsebene in Fall 1 ist vielfältig. Einen besonderen Stellenwert nehmen die Öffentlichkeitsarbeit und Teilnehmendengewinnung ein, die zugleich als zentrale Herausforderungen thematisiert werden. Eine zentrale Position kommt dabei dem Grundbildungszentrum und dem Kooperationsverbund zu, die in ihrem Leistungsangebot den Schwerpunkt darauf legen, ,die Angebote der Region öffentlich zu machen“ (2.1_Z.209). Insgesamt zeigt sich, dass die Akteure darauf fokussiert sind, „Vernetzung zu schaffen und Strukturentwicklung zu machen“ (1.2_Z.4-8). Das Bewusstsein dafür, dass ,Synergien schon da sind, die weitergetragen werden“" müssen (1.1_Z.180-181), ist ein Merkmal von Fall 1. Es gibt eine dichte Projektstruktur, die von der Vielfalt der aktiv eingebundenen Akteure lebt. Ein intensiver Austausch zwischen den „Bildungs- und Beratungsträgern der Region“ (1.2_Z.165-166) dient dazu, neue Projektideen zu erschließen und den Informationsfluss untereinander zu gewährleisten. Zentral ist das Grundbildungszentrum, das als „Clearingstelle“ (1.3_Z.36) fungiert:

Er hat quasi in diesen ganzen Kommunen Multiplikatoren- und Sensibilisierungsarbeit geleistet also ist überall hingefahren, hat überall mal bei den verschiedensten Akteuren Vortrag gehalten über was is'n Grundbildung überhaupt, was ist Alphabetisierung und was können wir überhaupt als Kommune oder wir gemeinsam als Grundbildungszentrum mit der Kommune zusammen machen (1.3_Z. 36-38).

Die Zusammenarbeit in der Region geschieht im Wesentlichen und dominierend in der Form der gemeinschaftlichen Handlungskoordination, die gekennzeichnet ist durch eine affektiv orientierte Zielfokussierung:

da ging es mehr um's große Ganze, wo wollen wir inhaltlich hin, wie müssen wir unseren Meilensteinplan abarbeiten? (1.2_Z.197-199).

Die gemeinsame Arbeit findet statt in Form von Austauschrunden, an denen sowohl die Dachorganisationen als auch Mitgliedsorganisationen beteiligt sind,

um auch die Bildungsakteure der Region zusammenzubringen, an einen Tisch zu bringen und ja quasi, dass nicht alle das Rad neu erfinden, dass nicht alle die gleichen Projekte, Projektanträge stellen, natürlich steuern wir das nicht, aber wir können die anderen fragen, was macht ihr denn? (1.2_Z.182-185).

Darüber hinaus dient die intensive Zusammenarbeit dazu, Öffentlichkeitsarbeit und Teilnehmendengewinnung in der Region zu gewährleisten. Dies geschieht in der Handlungskoordinationsform des Netzwerks, in dem der Umgang mit Ressourcen und der Austausch im Fokus stehen. Sowohl einzelne Organisationen als auch Netzwerke und Unternehmen nutzen die bewährten Kontakte, um eine Vermittlung von Teilnehmenden in passgenaue Angebote ermöglichen zu können: 
gibt vielleicht noch keine Alphabetisierungsangebote vor Ort, sondern man sieht Akteure, man sieht Ratsuchende, aber weiß noch nicht so genau, wie kriegen wir die von dort zu euch (1.3_Z.90).

Handlungskoordination in Form der Hierarchie hat eine besondere Relevanz, wenn es um Fragen der Finanzierung bzw. Weiterfinanzierung und neuer Projektakquise geht. Die politischen Akteure weisen eine Entscheidungsmacht mit den zur Verfügung stehenden Regelmechanismen durch die entsprechenden Förderinstrumente auf:

Das ist auch nur 'ne Projektförderung die beginnt und beendet wird nach drei Jahren sprich da sind wir irgendwie noch nicht in einer Regelsystematik drin mit zu unterstützen, [...] es könnten Regelinstrumente werden, die eben nicht projektabhängig sind, sondern sozusagen Gesetzesmäßig implementiert sind, ja? (1.1_Z.221-224)

Die Arbeit der Akteure ist nur in einer Zeitspanne (hier drei Jahre) strukturentwickelnd möglich ist und hat eine entsprechend kurze Halbwertszeit. Durch den bereits beschriebenen Fokus der Bildungseinrichtungen auf gemeinschaftliche und netzwerkliche Handlungskoordinationen untereinander entsteht allerdings eine Erweiterung des Handlungsspielraums:

zum Beispiel mit der Sozialdezernentin, die dann in die Stadt hineingewirkt hat und uns da Tore aufgestoßen hat, oder eben auch, dass wir von anderen Projekten wie [Projektname] mit den Kollegen dort auch eben kommunizieren, weil die ja die Arbeitnehmervertretungen sensibilisieren und wir dann sozusagen als Nachfolgeaktivität auch die Arbeitnehmer eben schulen könnten (1.1_Z.50-53).

Die starken netzwerklichen Formen der Handlungskoordination in der Region ermöglichen gewissermaßen ein Auffangen und Abflachen dieser starken hierarchischen Steuerung durch Projektfinanzierungen. Der Abhängigkeit durch die finanziellen Mittelgeber kann durch die starke Vernetzung des Feldes und dem Nutzbarmachen von vorhandenen Synergien mit Ausweichstrategien begegnet werden.

\subsubsection{Fall 2}

Fall 2 ist als intermediär verdichtetes organisationales Feld zu charakterisieren. Der Fall verfügt über kein Grundbildungszentrum, wie Fall 1. Der bundeslandübergreifende Kooperationsverbund wird von dem Landesverband der Volkshochschulen koordiniert. Aktiv sind darüber hinaus diverse Bildungsorganisationen für AoG in der Region. Der Fall ist gekennzeichnet durch viele Großstädte und Ballungsgebiete. Insgesamt ist ein breit aufgestelltes Projektspektrum zu verzeichnen, das hauptsächlich im Rahmen von Bundesmitteln, ESF- bzw. Landesmitteln gefördert wird.

In Fall 2 ist Netzwerkarbeit nicht nur das vorrangige Leistungsangebot, sondern Grundvoraussetzung, um Alphabetisierung und Grundbildung in der Region erfolgreich realisieren zu können. So gibt es regionale Zusammenschlüsse von Volkshochschulen, die sich mit dem Ziel zusammenfinden, einen Austausch über die Ge- 
winnung von Lehrenden und Angebotsformaten zu gewährleisten. Der Fokus liegt zudem auf einer in die Breite getragenen Öffentlichkeits- und Sensibilisierungsarbeit.

In Fall 2 ist der dominierende Modus der Handlungskoordination in der netzwerklichen Zusammenarbeit in einer gemeinsamen Projekt- und Angebotsplanung zu sehen:

dass eben dieses Netzwerken sehr wichtig ist [...] und dass es eben wichtig ist möglichst viele Akteure zusammenzubringen in dem Thema und gemeinsam Sachen zu erarbeiten (2.1_Z.15-18) und sich entsprechend daraus ,die Haupttätigkeit“" ergibt (2.2_Z.66).

Der besondere Mehrwert netzwerklicher Zusammenarbeit besteht in der Bündelung von Ressourcen und dem daraus folgenden Wissen, sich jederzeit an professionelle Ansprechpartnerinnen und Ansprechpartner wenden zu können:

wenn man da irgendwie mal ne Expertise braucht, weiß man ok, an die kann man sich wenden, die kommen dann gerne und erzählen was zu nem bestimmten Thema, also da gibt es wirklich sehr sehr viele Expertise (2.2_Z.246-248).

Für lokale Volkshochschulen ist die kommunale Politik ein relevanter Akteur. Beispielsweise hat eine Kommune

[...] sich entschieden jetzt mit der Entgeltbefreiung diese Kurse jetzt kostenfrei anzubieten [...] (2.2_Z.16).

Unter anderem wurde die Debatte der Entgeltbefreiung veranlasst, um

den Abgeordneten des Rats der Stadt halt auch ja ne Sensibilität dafür zu schaffen und denen mal aufzuzeigen was letztlich hinter einer fehlenden Schriftsprachkompetenz alles dahintersteckt (2.2_Z.16).

So zeigt sich in Fall 2 insgesamt eine starke Zusammenarbeit mit politischen Akteuren. Dies löst sich jedoch im Gegensatz zu den Strategien der Akteure in Fall 1 nicht in horizontaler netzwerklicher Handlungskoordination auf, sondern in individuellen Beratungs- und Informationsleistungen des Kooperationsverbunds und der Bildungsorganisationen:

[...] , dass ich dann wiederum dem Ministerium also meinem Mittelgeber, sag ich mal, dem Fördermittelgeber berichten kann, was sind denn so die Bedarfe aus der Praxis, wo läuft es gut, wo läuft es nicht so gut und dass man da eigentlich in so einem ständigen Austausch steht (2.1_Z.23-28).

Entsprechend geht es den Akteuren der Bildungseinrichtungen darum, Strukturen aufzubauen und zu verstetigen, indem sie längerfristige Förderungen verhandeln. Der besondere Bedarf entsteht bei der Verlässlichkeit und Ansprechbarkeit von Partnern im Feld, die nötigenfalls aufgrund von auslaufender Finanzierung für gemeinschaftliches und netzwerkliches Handeln nicht mehr zur Verfügung stehen.

$\mathrm{Ne}$, man braucht schon irgendwas verlässliches oder so mindestens fünf Jahre bräuchte man eigentlich schon, um zu sehen, ok, da baut sich was auf, ne, da 
ist jemand, der ist verlässlich da, der hat auch Geld. Also das ist, man muss da auch einfach mal über Geld sprechen (2.1_Z.331-333).

Diese Konstellation im Zusammenhang mit den politischen Akteuren, die über die klare Ansprache und Aushandlung von Finanzierungsfragen besteht, hat jedoch keinen Einfluss auf die weiterhin hierarchische Handlungskoordination. Sichtbar wird diese:

wenn bestimmte Institutionen keine Förderung mehr bekommen für das Thema, ne dann kann es auch einfach sein, dass da der ganze Bereich mal eingestampft wird (2.1_Z.324-326).

\subsubsection{Fall 3}

Fall 3 ist gekennzeichnet durch eine geringere Dichte des organisationalen Feldes in der AoG. Die zentralen und umsetzenden Akteure hinsichtlich AoG-bezogener Angebote stellen die Volkshochschulen dar. Vereinzelt gibt es landes- oder bundesfinanzierte Projekte. Die Leistungsebene in Fall 3 ist fokussiert auf Angebote für Unternehmen.

Es zeigt sich dabei, dass diese Leistungen ein breites Spektrum umfassen: Neben der Qualifizierung von unternehmensinternen Bildungsberatern, die sowohl als offene Qualifizierungen als auch in Form von Inhouse-Schulungen stattfinden, beinhalten die Leistungen zusätzlich die kontinuierliche Begleitung, Motivation und Versorgung mit Informationen der Unternehmen.

Entsprechend dieses Leistungsspektrums zeigt sich in Fall 3 eine starke Zusammenarbeit mit Unternehmen, für die sie Angebote entwickeln und durchführen. Hier lässt sich - auch im Vergleich zu den anderen Fällen - eine Besonderheit markieren: In Zusammenarbeit mit den Unternehmen haben die Bildungsorganisationen des Falls 3 die zunächst marktförmigen Handlungskoordinationen in eine eher netzwerkliche Zusammenarbeit transformiert. So wird das Vorgehen in der Ansprache der Unternehmen in der Vergangenheit mit „Kaltakquise“ (3_Z.206) beschrieben, bei der nach vorheriger Beobachtung des Feldes und konkreter Benennung der relevanten Ansprechpartnerinnen und Ansprechpartner die Handelnden ,einfach geschaut haben, ob das ankommt oder nicht“"(3_Z.206).

Die Strategie hat sich inzwischen insofern verändert, als Kaltakquise nicht mehr notwendig ist und die Betriebe nun auf die Anbieter zukommen. Eine besondere Rolle spielt da der kooperative Zugang zu betriebsinternen Weiterbildungsstrukturen, der eher in großen Unternehmen möglich ist:

eher sagen, wir erreichen größere Betriebe, weil da einfach auch die interne Vernetzung und interne Strukturen vorhanden sind, also Betriebsrat zum Beispiel, Weiterbildungsabteilung, es gibt ne Personalabteilung, es gibt Wege, dass also wie man für Beschäftigte bestimmte Angebote vorbereitet und was, was man anbieten kann im Betrieb (3_Z.236-238).

Über diese starke Zusammenarbeit im Modus des Netzwerks sichern und erweitern sich die Akteure des Falls 3 das Leistungsspektrum. 
Darüber hinaus erweist sich die starke Vernetzung mit anderen Bildungsträgern als besonders ertragreich. Dabei werden Verweisungsstrukturen in Bezug auf die Teilnehmenden gebildet und zu diesem Zweck persönliche Kontakte aufgebaut und gehalten:

Wenn es aber darum geht, die eigentliche Zielgruppe also da tatsächlich zu verweisen, dann hat sich das immer bewährt, dass wir im persönlichen $\mathrm{Ge}$ spräch mit den einzelnen Trägern sind, also mit den Personen und so dass es ein schneller kurzer Weg ist (3_Z.276-278).

Wichtig ist dabei, dass schnelle Kommunikationswege für die kurzfristige Angebotssuche für Teilnehmende möglich sind:

Also wir greifen dann zum Telefon und sagen ,hier unser [Lerner] hat jetzt eine Person, die macht sich vielleicht auf den Weg, “würde das passen, könntest du nicht ein Erstgespräch führen und schauen, ob das, ob das passt? (3._Z.278-280).

Diese netzwerklichen Strukturen sind für die Bildungsträger in der Region von Vorteil, indem sie das Angebotsportfolio erweitern:

ok wir bieten zwar das und das an, aber uns fehlt ein anderer Bereich (3._Z.175-176).

Im Zusammenhang mit dem Umgang unsicherer Finanzierungsituationen zeigt sich ein interessanter Aspekt in der Handlungskoordinationsform der Hierarchie: Politische Akteure sind über die über die Finanzierung im Zusammenhang mit dem Fördergeber BMBF relevant. So wird diskutiert, was bei Auslaufen der Finanzierung mit den aufgebauten Strukturen geschieht. Es gibt dabei gewisse Routinen im Umgang mit dem Auslaufen von Projekten und dem Auffangen von Strukturen ,so dass das im Grunde zu unserem täglichen Geschäft gehört“ (3_Z.427). Anders als in den Fällen 1 und 2 durch gemeinschaftliches, netzwerkliches Handeln oder auch durch Beratung der politischen Akteure, werden hier keine strategischen Bemühungen gegen das Auslaufen von Finanzierung deutlich.

\subsection{Governance-Regimes in der AoG}

Nach der Beschreibung der in der Studie untersuchten Fälle mit der jeweils spezifischen Dichte des organisationalen Feldes und die verschiedenen Formen der Handlungskoordination folgt hier eine kurze Zusammenfassung und ein Vergleich der Fälle. Darauf aufbauend lassen sich erste Einblicke in Governance-Regimes in der AoG geben.

Die umfassenden pädagogischen Leistungen in den Fällen sind auf die forcierte und systematische Vernetzung der Weiterbildungsakteure in der Region zurückzuführen.

Dies wird insbesondere in Fall 1 und Fall 2 deutlich. Das Leistungsspektrum bewegt sich zwischen einer in der Region gemeinschaftlich forcierten Öffentlichkeitsarbeit und Teilnehmendengewinnung (bei der die Akteure dann von den entwickelten Verweisstrukturen direkt profitieren) und der in netzwerklichen Zusammenhängen 
geteilten Angebots- und Projektplanung. Fall 3 weist dabei im Hinblick auf die Leistungsstruktur eine geringere Reichweite auf, da hier wesentlich lokale Angebote für Teilnehmende in Unternehmen und weniger Leistungen in der Region entwickelt werden. In allen drei Regionen ist das Leistungsspektrum jedoch vielfältig und differenziert.

Handlungskoordinationen weisen je nach Fall verschiedene Ausprägungen auf. Dominant zeigt sich in allen Fällen hierarchische Handlungskoordination in der Abhängigkeit von finanziellen Mitteln, die für die Bildungsorganisationen über den Auf- und Abbau von Strukturen bestimmen. Fall 1 zeigt deutlich ausgeprägtere Varianten im Umgang mit hierarchischer Handlungskoordination. Die Akteure auf der Ebene der Bildungseinrichtungen sind durch stark ausgebaute netzwerkliche und gemeinschaftliche Zusammenhänge in der Lage, den Akteuren mit absoluter Entscheidungsmacht $\mathrm{zu}$ begegnen und sie weisen eine gewisse Eigenständigkeit auf, wenn es darum geht, finanzielle Ressourcen über herkömmliche Strukturen hinaus zu akquirieren. In Fall 3 haben die Akteure im Hinblick auf die Unsicherheiten in der Finanzierung einen routinierten Umgang. Insgesamt werden gemeinschaftliche und netzwerkliche Handlungskoordination mit bekannten Verweisstrukturen zur gelingenden Teilnehmendengewinnung nutzbar gemacht. Hierbei ist eine ausgeprägte Akteurslandschaft wie in Fall 1 förderlich. Gemeinschaftliche Handlungskoordination, die im Kern über eine geteilte normative Zielvorstellung der Arbeit im Kontext von AoG funktioniert, ist insgesamt von großer Bedeutung, indem Austausch (auch überregional) möglich ist und Themen erarbeitet werden. Die Spezifik des Falls 3 ist eine Entwicklung von marktförmiger zu netzwerklicher Handlungskoordination zwischen Bildungseinrichtungen und Unternehmen.

Betrachtet man die vorherrschenden Akteurskonstellationen und Handlungskoordinationsformen im Feld der AoG im Gesamten, lassen sich gewisse Einblicke in Governance-Regimes als ein Zusammenwirken von verschiedenen Mechanismen (Kussau und Brüsemeister 2007, S. 41; Schimank 2007, S. 203) ermöglichen.

Das vorherrschende Governance-Regime besteht in Abhängigkeit zu den strukturellen Bedingungen in den jeweiligen Regionen, die zum einen variierende organisationale Dichten über vielfältige Akteurskonstellationen verzeichnen und zum anderen in verschiedenen Formen der Handlungskoordinationen Zusammenarbeit ermöglichen. So zeigen sich in der jeweiligen Dichte der organisationalen Felder von AoG typische Formen der Handlungskoordination, die sich zwischen netzwerklichen, hierarchischen und marktförmigen Koordinationsformen bewegen. Hierarchie hingegen hat auch über die Unterscheidung der Strukturierungsgrade gleichermaßen Einfluss, indem politische Akteure über Finanzierung entscheiden und dies die Akteure der Bildungseinrichtungen direkt beeinflusst. Die organisationalen Felder, die sich nach Dichtegraden unterscheiden lassen, haben dann jeweils typische Koordinationsformen, um der Finanzierungssituation $\mathrm{zu}$ begegnen. Im Hinblick auf pädagogische Leistungserbringung hat dies dann Auswirkungen. So sind im stark verdichteten organisationalen Feld des Falls 1 durch starke netzwerkliche Zusammenarbeit Handlungsspielräume erweitert worden. Im intermediär verdichteten Feld des Falls 2 führen netzwerkliche und gemeinschaftliche Handlungskoordinationen zu einer Sichtbarmachung der Leistung und insbesondere zur Gewinnung von Teilnehmenden. Das gering verdichtete Feld des Falls 3 kann sich über starke Zu- 
sammenarbeit mit Unternehmen Leistung sichern und stärkt durch überregionale gemeinschaftliche Zusammenarbeit die Entwicklung von Themen und Vielfalt der Leistung.

Damit erweist sich Fragilität der Finanzierung als ein dominantes Kennzeichen aller hier untersuchten Fälle. In unterschiedlichen Spielarten von Akteurskonstellationen und Formen der Handlungskoordination sichern die Akteure die jeweiligen pädagogischen Leistungen ab. Interessant ist dabei, dass die Ausprägungen der Leistungsstruktur sich nur graduell unterscheiden, insgesamt jedoch vielfältig sind. Unterschiede entstehen lediglich in der Reichweite der Angebote und der Stärke der Verweisstrukturen, die sich günstig auf die Stabilität der Leistungsstruktur ausprägen.

\section{Fazit}

Der vorliegende Beitrag analysiert unter Rückgriff auf die Perspektive der Educational Governance und des Neo-Institutionalismus, welche organisationalen Strukturen sich aktuell in der AoG erkennen lassen und welche Formen der Handlungskoordination welche pädagogischen Leistungen ermöglichen.

Mit Blick auf die organisationalen Strukturen lässt sich festhalten, dass AoG im bundesweiten Überblick über organisationale Strukturen unterschiedlicher Dichte verfügt. Dabei konnten drei Idealtypen identifiziert werden, anhand derer in einer qualitativen multiple case study sodann Formen der Handlungskoordination und Leistungen in Regionen mit unterschiedlicher organisationaler Dichte fokussiert wurden.

Insgesamt lässt sich festhalten, dass die Akteure im Feld - hier maßgeblich die Bildungseinrichtungen, Volkshochschulen und aktiven Projekte im Bereich der Alphabetisierung und Grundbildung - in manifestierten, netzwerklichen und gemeinschaftlichen Handlungskoordinationsformen agieren. Je manifestierter die Handlungskoordinationsformen sind und je klarer die Zuständigkeiten und Verweisstrukturen innerhalb der Akteurskonstellationen, desto handlungs- und leistungsfähiger scheinen die Akteure. Es wird zudem deutlich, dass sich die verdichtete Struktur positiv auf Aspekte wie die gemeinschaftliche Öffentlichkeitsarbeit sowie auf Teilnehmendengewinnung auswirken. Bei geringerer organisationaler Dichte ist die Reichweite der entsprechenden Aktivitäten geringer. Insgesamt zeigen sich in den Fällen unterschiedliche Muster der Handlungskoordination, die allesamt ein vielfältiges und differenziertes Leistungsspektrum eröffnen.

Abschließend sollen die Befunde nochmals mit Blick auf Praxisrelevanz, Bildungspolitik und den Beitrag zur wissenschaftlichen Debatte eingeordnet werden. 
Mit Blick auf die praktische Nutzung der Befunde ist auf Ergebnisse in Workshops mit Expertinnen und Experten in der AoG sowie auf bereits publizierte Handlungsempfehlungen zur Entwicklung von Perspektiven des Feldes zu verweisen. ${ }^{4}$

In bildungspolitischer Hinsicht legt sich die Förderung der Vernetzung und die Steigerung von organisationaler Dichte nahe, da es so gelingt, die Reichweite innerhalb der Region zu erhöhen. Bei der Steigerung der organisationalen Dichte scheint insbesondere die Einbindung von Sozialpartnern und der Arbeitsverwaltung sinnvoll, um auch Optionen regelhafter Finanzierung sukzessive zu erweitern. Wichtig insgesamt ist jedoch die Sicherung der Vielfalt durch die Einbindung der arbeitsmarktbezogenen Weiterbildungslandschaft.

Schließlich soll auch der Beitrag zur wissenschaftlichen Debatte abschließend gewürdigt werden. Der Aufsatz leistet einerseits einen Beitrag zur Struktur- und Organisationsforschung in der Erwachsenenbildung, indem er Handlungskoordination in fragilen Organisationsstrukturen der Erwachsenenbildung analysiert. Mit der Verschränkung von Educational Governance und Neo-Institutionalismus in der Erwachsenenbildungsforschung gelingt es, wie gezeigt, sehr gut und auf produktive Weise, fragile Bereiche wie AoG mit Blick auf Akteurskonstellationen, Handlungskoordination und pädagogische Leistung zu untersuchen. Als weitere Forschungsperspektive lässt sich zum einen ein Vergleich mit anderen fragilen Bereichen der Weiterbildung, wie etwa kulturelle Bildung, mit Blick auf Handlungskoordination und Leistungsspektrum festmachen. Zum anderen wäre auch ein internationaler Vergleich denkbar.

Des Weiteren konnte auch ein Beitrag zur Forschung im Feld der arbeitsorientierten Grundbildung geleistet werden. Dabei konnten Aspekte der organisationalen Struktur, der Steuerung sowie der Handlungskoordination und der pädagogischen Leistung erstmalig analysiert werden.

In den Blick anschließender Forschung sollten Möglichkeiten und Bedingungen der Verstetigung von arbeitsorientierter Grundbildung geraten.

Funding Open Access funding enabled and organized by Projekt DEAL.

Open Access Dieser Artikel wird unter der Creative Commons Namensnennung 4.0 International Lizenz veröffentlicht, welche die Nutzung, Vervielfältigung, Bearbeitung, Verbreitung und Wiedergabe in jeglichem Medium und Format erlaubt, sofern Sie den/die ursprünglichen Autor(en) und die Quelle ordnungsgemäß nennen, einen Link zur Creative Commons Lizenz beifügen und angeben, ob Änderungen vorgenommen wurden.

Die in diesem Artikel enthaltenen Bilder und sonstiges Drittmaterial unterliegen ebenfalls der genannten Creative Commons Lizenz, sofern sich aus der Abbildungslegende nichts anderes ergibt. Sofern das betreffende Material nicht unter der genannten Creative Commons Lizenz steht und die betreffende Handlung nicht nach gesetzlichen Vorschriften erlaubt ist, ist für die oben aufgeführten Weiterverwendungen des Materials die Einwilligung des jeweiligen Rechteinhabers einzuholen.

Weitere Details zur Lizenz entnehmen Sie bitte der Lizenzinformation auf http://creativecommons.org/ licenses/by/4.0/deed.de.

\footnotetext{
4 Es wurden Handlungsempfehlungen für die Öffentlichkeitsarbeit, für die Forschung zum Thema, aber auch für die Entwicklung von Lernangeboten etc. entwickelt. Diese sind abrufbar unter: https://www. grundbildung-wirkt.de/cms/wp-content/uploads/2020/08/AoG-Überblick-und-Perspektiventwicklung. pdf.
} 


\section{Literatur}

Altrichter, H. (2015). Governance - Steuerung und Handlungskoordination bei der Transformation von Bildungssystemen. In H. J. Abs, T. Brüsemeister, M. Schemmann \& J. Wissinger (Hrsg.), Governance im Bildungssystem: Analysen zur Mehrebenenperspektive, Steuerung und Koordination (S. 21-63). Wiesbaden: Springer. https://link.springer.com/content/pdf/10.1007\%2F978-3-658-06523-2.pdf. Zugegriffen: 07.04.2021.

Altrichter, H., Brüsemeister, T., \& Wissinger, J. (Hrsg.). (2007). Educational Governance: Handlungskoordination und Steuerung im Bildungssystem (1. Aufl.). Wiesbaden: Springer.

Benz, A., \& Dose, N. (2010). Governance - Regieren in komplexen Regelsystemen. Eine Einfuihrung (2. Aufl.). Wiesbaden: Springer.

BMBF - Bundesministerium für Bildung und Forschung, \& Kultusministerkonferenz (2016). Grundsatzpapier zur Nationalen Dekade für Alphabetisierung und Grundbildung. 2016-2026. Den funktionalen Analphabetismus in Deutschland verringern und das Grundbildungsniveau erhöhen. Berlin. https:// www.bibb.de/dokumente/pdf/a33_grundsatzpapier_nationale_dekade.pdf. Zugegriffen: 7. Apr. 2021.

DiMaggio, P.J., \& Powell, W.W. (2009). Das „stahlharte Gehäuse“ neu betrachtet: Institutioneller Isomorphismus und kollektive Rationalität in organisationalen Feldern. In S. Koch \& M. Schemmann (Hrsg.), Neo-Institutionalismus in der Erziehungswissenschaft (S. 57-84). Wiesbaden: Springer.

Euringer, C. (2016). Das Grundbildungsverständnis der öffentlichen Bildungsverwaltung: Definitionen, Interessen und Machtverhältnisse. Bielefeld: W. Bertelsmann.

Faulstich, P., \& Haberzeth, E. (2015). Weiterbildungspolitik. In J. Dinkelaker \& A. von Hippel (Hrsg.), Erwachsenenbildung in Grundbegriffen (1. Aufl. S. 263-271). Stuttgart: Kohlhammer.

Hartz, S., \& Schrader, J. (2008). Steuerung und Organisation in der Weiterbildung - ein vernachlässigtes Thema? In S. Hartz \& J. Schrader (Hrsg.), Steuerung und Organisation in der Weiterbildung (S. 9-30). Bad Heilbrunn: Klinkhardt.

Herbrechter, D., \& Schemmann, M. (2019). Educational Governance und Neo-Institutionalismus in der Weiterbildungsforschung. In R. Langer \& T. Brüsemeister (Hrsg.), Handbuch Educational Governance Theorien, Educational Governance (Bd. 43, S. 181-199). Wiesbaden: Springer. https://link. springer.com/content/pdf/10.1007\%2F978-3-658-22237-6_9.pdf. Zugegriffen: 07.04.2021.

Hof, C., Ludwig, J., \& Schäffer, B. (Hrsg.). (2011). Steuerung - Regulation - Gestaltung: GovernanceProzesse in der Erwachsenenbildung zwischen Struktur und Handlung; Dokumentation der Jahrestagung der Sektion Erwachsenenbildung der Deutschen Gesellschaft für Erziehungswissenschaft. Baltmannsweiler: Schneider.

Koch, S., \& Schemmann, M. (2009). Neo-Institutionalismus in der Erziehungswissenschaft: Grundlegende Texte und empirische Studien. Wiesbaden: Springer.

Koller, J., Klinkhammer, D., \& Schemmann, M. (Hrsg.). (2020). Arbeitsorientierte Grundbildung und Alphabetisierung. Institutionalisierung, Professionalisierung und Effekte der Teilnahme. Bielefeld: wbv.

Kowalczyk, K., Neureiter, M., Pabst, A., \& Zeuner, C. (2016). Evaluation des BMBF-Förderschwerpunkts „Arbeitsplatzorientierte Alphabetisierung und Grundbildung Erwachsener“. Abschlussbericht. Berlin: BMBF. https://www.hsu-hh.de/eb/wp-content/uploads/sites/662/2020/03/AbschlussberichtArbeitsplatzorientierte-Alphabetisierung-und-Grundbildung-Erwachsener_mit-Anhang.pdf. Zugegriffen: 07.04.2021

Krippendorff, K. (2004). Reliability in content analysis: some common misconceptions and recommendations. Human Communication Research, 30(3), 411-433.

Kussau, J., \& Brüsemeister, T. (2007). Educational Governance: Zur Analyse der Handlungskoordination im Mehrebenensystem der Schule. In H. Altrichter, T. Brüsemeister \& J. Wissinger (Hrsg.), Educational Governance: Handlungskoordination und Steuerung im Bildungssystem 1. Aufl. Wiesbaden: Springer.

Lernende Region - Netzwerk Köln e.V. (2021). Grundbildung in der Arbeitswelt gestalten. Praxisbeispiele, Gelingensbedingungen und Perspektiven. Bielefeld: wbv Publikation.

Maag Merki, K., \& Altrichter, H. (2010). Empirische Erforschung schulischer Governance. Eine Zwischenbilanz und offene Forschungsfragen. In H. Altrichter \& K. Maag Merki (Hrsg.), Handbuch Neue Steuerung im Schulsystem 1. Aufl. (Bd. 7, S. 403-408). Wiesbaden: Springer. https://doi.org/ 10.1007/978-3-531-92245-4_16.

Mayntz, R. (1998). New Challenges to Governance Theory. In R. Mayntz (Hrsg.), Über Governance. Institutionen und Prozesse politischer Regelung (S. 13-27). Frankfurt a.M.: Campus.

Mayring, P. (2015). Qualitative Inhaltsanalyse: Grundlagen und Techniken (12. Aufl.). Weinheim: Beltz. 
Schemmann, M. (2020). „Und sie bewegt sich doch“ - Neue Steuerung und Governance in der öffentlichen Weiterbildung. In I. van Ackeren, H. Bremer, F. Kessl, H.C. Koller, N. Pfaff, C. Rotter, D. Klein \& U. Salaschek (Hrsg.), Bewegungen: Beiträge zum 26. Kongress der Deutschen Gesellschaft für Erziehungswissenschaft (1. Aufl. S. 391-403). Opladen, Berlin, Toronto: Budrich. https://doi.org/10. 2307/j.ctv10h9fjc.

Schimank, U. (2007). Die Governance-Perspektive: Analytisches Potenzial und anstehende konzeptionelle Fragen. In Educational Govnance. Handlungskoordination und Steuerung in Bildungssystemen (1. Aufl. S. 231-260). Wiesbaden: Springer.

Schrader, J. (2011). Struktur und Wandel der Weiterbildung. Bielefeld: W. Bertelsmann.

Schroeder, J. (2016). Arbeitsplatzorientierte Grundbildung. In C. Löffler \& J. Korfkamp (Hrsg.), Handbuch zur Alphabetisierung und Grundbildung Erwachsener (S. 237-249). Münster: Waxmann.

Schwarz, S. (2021). Arbeitsorientierte Grundbildung: Definition, Überblick und Einordnung. In Lernende Region - Netzwerk Köln e.V. (Hrsg.), Grundbildung in der Arbeitswelt gestalten. Praxisbeispiele, Gelingensbedingungen und Perspektiven (S. 17-23). Bielefeld: wbv Publikation.

Yin, R. K. (2009). Case study research. Design and methods (4. Aufl.). Bd. 5. Kalifornien: Sage.

Publisher's Note Springer Nature remains neutral with regard to jurisdictional claims in published maps and institutional affiliations. 